\title{
Finite element analysis of multi-hole extrusion of aluminum-alloy tubes
}

\author{
Fuh-Kuo Chen $^{a, *}$, Wen-Chan Chuang ${ }^{a}$, Shan Torng ${ }^{b}$ \\ a Department of Mechanical Engineering, National Taiwan University, Taipei, Taiwan, ROC \\ b Chung-Shan Institute of Science \& Technology, Lung-Tan, Tao-Yuan, Taiwan, ROC
}

\section{A R T I C L E I N F O}

Keywords:

Multi-hole extrusion

Balanced flow

Eccentricity index

Finite element analysis

\begin{abstract}
A B S T R A C T
The multi-hole extrusion of aluminum-alloy tubes was examined in the present study using both the finite element analysis and the experimental approach. The finite element analysis was first validated qualitatively and quantitatively by the experimental data obtained from the single-hole extrusion process. The effects of the process parameters, such as extrusion temperature, extrusion speed, dimensions of billet, and location of holes on the extrusion load and the shape of extruded tubes were then studied by the finite element analysis. The finite element analysis reveals that the most crucial process parameter is the number of holes and their locations on the extrusion die. It is also found that a uniform deformation of mandrel and a balanced flow at the exit of extrusion die could be achieved if the position of holes is near the centroid of the die area. An expression of the eccentricity of the holes was defined by a ratio. An eccentricity ratio that represents an optimum position of the holes was then developed for the two-hole extrusion of aluminum-alloy tubes to avoid any bending or curvature of the extruded product. The developed approach could be also extended to other multi-hole extrusion of more than two tubes and help the die engineer to design a more productive extrusion process.
\end{abstract}

(c) 2007 Elsevier B.V. All rights reserved.

\section{Introduction}

The single-hole extrusion process is commonly adopted in industry to produce circular tubes. However, in order to increase the productivity, the multi-hole extrusion process is also considered by the practising engineers to design an efficient extrusion tooling. Most multi-hole extrusion processes are applied to produce seamed hollow section tubes using the porthole die (Kim et al., 2002; Jo et al., 2002), in which the divided bars are welded in the welding chamber before being extruded to the exit. Compared to the complex construction of the die set in the porthole die extrusion, the conventional indirect extrusion is much simpler to be designed. For producing seamless tubes, an internal piercer with mandrel is used in the indirect extrusion, as shown in Fig. 1. The main advantage of indirect extrusion is the absence of friction between the billet and the container, resulting in a lower extrusion load and more uniform material flow.

In the multi-hole extrusion, most research efforts are usually aimed to search for an optimum die design to achieve a balanced flow at the die exit to avoid any bending of the extruded tubes. Analytical approach, such as upper-bound method, has been applied by many researchers to study the effect of the process variables on the extrusion pressure and extruded product shape (Ulysse and Johnson, 1998). The finite element analysis was also applied to examine the complex material flow in the flat-die hot extrusion process (Lee et al., 2002; Lee and Im, 2002) and in the extrusion for various num-

\footnotetext{
* Corresponding author. Tel.: +886 23366 2701; fax: +886 223631755. E-mail address: fkchen@ntu.edu.tw (F.-K. Chen). 


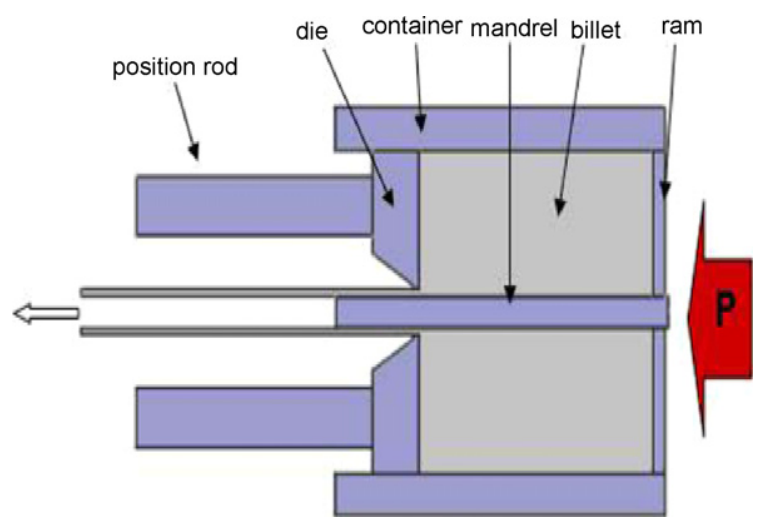

Fig. 1 - An indirect extrusion die set.

bers and locations of the holes (Peng and Sheppard, 2004). The effects of process parameters involved in the multi-hole extrusion were investigated by researchers using the finite element analysis as well (Li et al., 2004). Several of the process parameters involved are: the extrusion temperature, the extrusion speed, the friction, the extrusion reduction ratio, and the eccentricity of holes. It is then necessary to build up the relations of the interactive influence among these process parameters to help the practicing engineers to design effective dies.

In the present study, both the experimental approach and the finite element analysis were employed to study the effects of the process parameters on the multi-hole extrusion of aluminum-alloy A7075 tubes using the indirect extrusion process. The finite element model was first established for the single-hole extrusion and validated by the actual extrusion processes. The influences of the process parameters on the product shape of the extruded tubes were then investigated using the finite element simulations. Based on the finite element analysis, an optimum two-hole extrusion process that yields sound products without any curvature in the extruded tubes was developed.

\section{Single-hole extrusion}

Since the single-hole extrusion process is commonly adopted in industry to produce aluminum-alloy tubes, the production data is easily obtained from the actual extrusion processes. Therefore, the finite element analysis was first performed to study the effects of the process parameters on the extrusion load and exit temperature, and the simulation results could be validated by the production data. Two production tubes were selected. One is with $65 \mathrm{~mm}$ in outside diameter and $4.3 \mathrm{~mm}$ in tube thickness, the other being $61 \mathrm{~mm}$ in outside diameter and $3.0 \mathrm{~mm}$ in tube thickness. The former is denoted by Atube and the later is B-tube. The CAD software CATIA V5R10 was used to build the geometries of tooling and billet. The CAD model was then transferred to the finite element program DEFORM to establish the finite element mesh. In the finite element simulations, the tooling is treated as rigid body and only the billet is deformable. In order to save the computation time, the minimum billet length used for the simulation was first determined by comparing the simulation results obtained from various billets with different lengths. The finite element analysis reveals that a billet with $100 \mathrm{~mm}$ in length is long enough to render a reasonable simulation result.

The material properties of A7075 aluminum-alloy given in the DEFORM database was used for the simulations. The billet diameter is $203.2 \mathrm{~mm}$, and the initial extrusion temperature varies from $360^{\circ} \mathrm{C}$ to $400^{\circ} \mathrm{C}$. The extrusion speeds ranging from $0.3 \mathrm{~mm} / \mathrm{s}$ to $1.2 \mathrm{~mm} / \mathrm{s}$ for the A-tube and from $0.2 \mathrm{~mm} / \mathrm{s}$ to $0.8 \mathrm{~mm} / \mathrm{s}$ for the B-tube were adopted. In order to examine the effect of friction, extrusions with various friction factors (m) of $0.1,0.3$ and 0.5 were performed.

As for the experiments, a 1500 ton extrusion press was used to extrude both the A-tube and the B-tube. During the extrusion process, the extrusion load and the exit temperature can be measured. The extrusion experiments with the use of the same process parameters as those adopted in the finite element simulations were conducted, and the experimental data were compared with the finite element simulation results. Both the finite element simulations and the extrusion experiments are conducted for the A-tube and the B-tube, but only the results of the extrusion of A-tube are discussed hereafter to limit the length of this paper.

The effect of friction on the extrusion load was examined first. Fig. 2 shows the extrusion loads obtained from the finite element simulations with various friction factors for the extrusion of A-tube at initial temperature of $360^{\circ} \mathrm{C}$. It is clearly seen in Fig. 2 that the extrusion loads are almost the same as each other. It confirms that the effect of friction on the indirect extrusion is not significant. The extrusion loads for various initial forming temperatures obtained from the finite element simulation results and experiments for A-tube are compared. Both the finite element simulation results and the experimental data reveal that the extrusion load decreases as the forming temperature becomes higher due to the lower flow stress of the billet at higher temperature. It is also noticed that the extrusion loads obtained from the experiments are larger than those predicted by the finite element simulations. However, the differences are not significant. The temperatures at the exit of extrusion die are compared as well and the results are shown in Fig. 3. It is seen in Fig. 3 that both the finite element simulation results and the experimental data agree very well, though the measured temperatures are insignificantly lower. The exit temperatures resulted from various extrusion

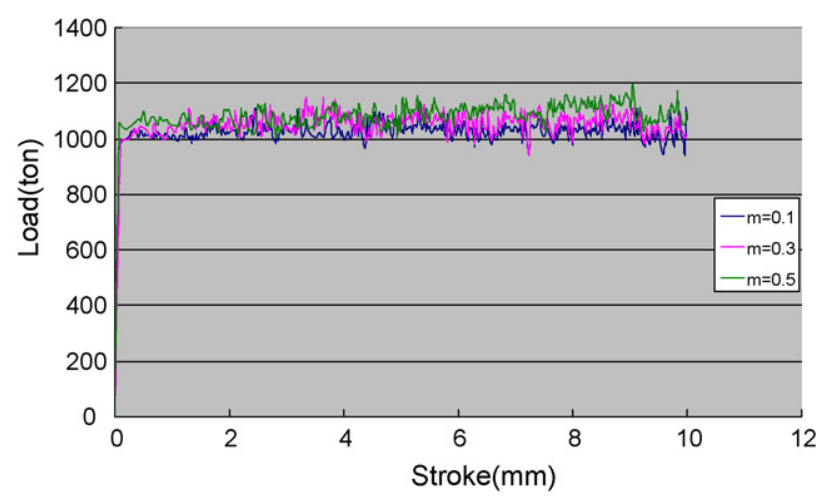

Fig. 2 - Extrusion loads for various friction factors (single-hole). 


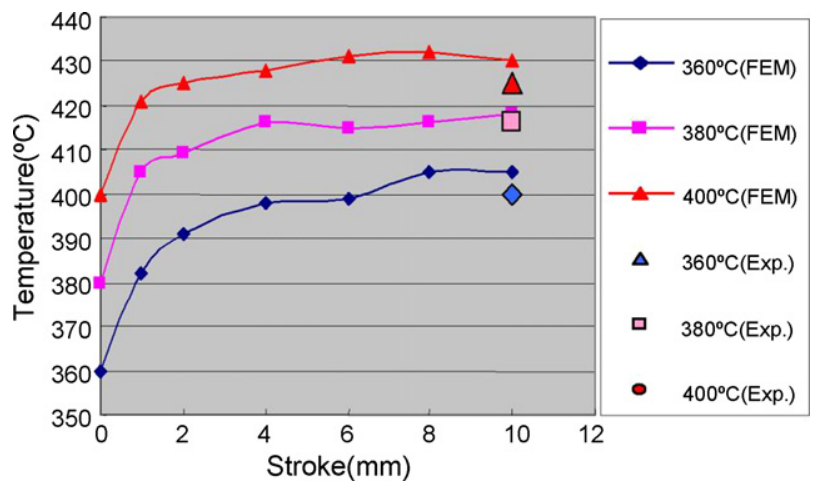

Fig. 3 - Exit temperatures for various initial extrusion temperatures (single-hole).

speeds also show a good agreement between the finite element simulation results and the experimental data. Thus, the accuracy of the finite element simulations is confirmed by the experimental data.

\section{Multi-hole extrusion}

The multi-hole extrusion of two tubes, three tubes, and four tubes were studied by the finite element analysis. The locations of the holes for each extrusion die set are displayed in Fig. 4(a-c), respectively. Due to symmetry, only the shaded areas shown in Fig. $4(\mathrm{a}-\mathrm{c})$ are used for the finite element simulations. The finite element model was built following the same procedure as that adopted for the single-hole extrusion. A larger billet of $254 \mathrm{~mm}$ in outside diameter was used for extrusion. Various initial forming temperatures and extrusion speeds were adopted as process variables to examine their effects on the extruded products, except that the friction factor of 0.3 is used for all simulations.

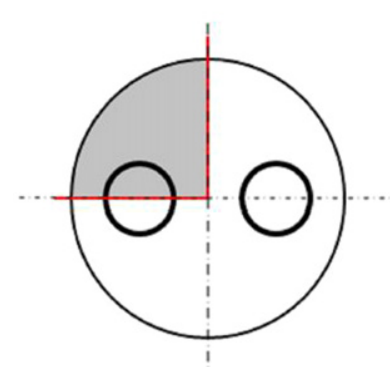

(a) two-hole

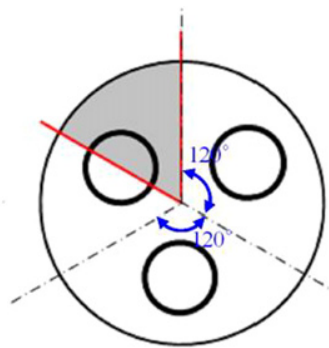

(b) three-hole

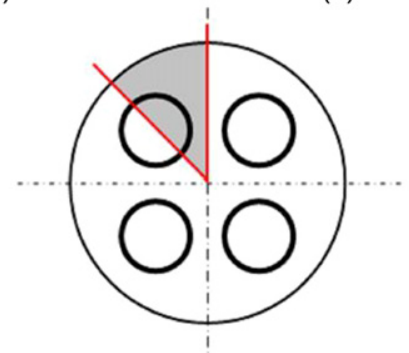

(c) four-hole

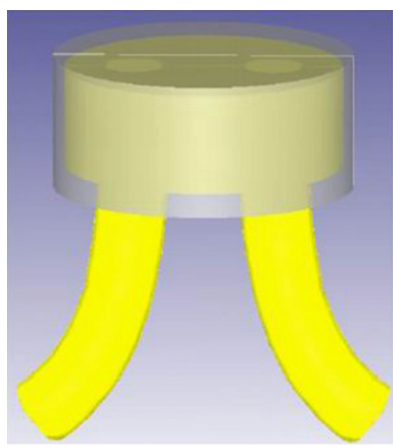

(a) two-hole

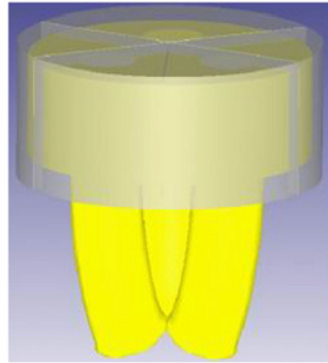

(b) three-hole

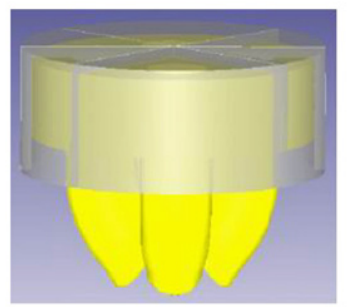

(c) four-hole

Fig. 5 - Extruded tubes with curvatures.

Fig. $5(\mathrm{a}-\mathrm{c})$ shows the extruded tubes at the exit of the extrusion dies for two-hole, three-hole, and four-hole, respectively. It is seen in these figures that the extruded tubes bear curvatures as they leave the extrusion dies. It is also noted that the extruded tubes bend outward in the two-hole extrusion; while the extruded tubes bend inward in both three-hole and four-hole extrusion processes. The curved tubes are due to unbalanced material flow at the exit of extrusion dies. The possible reasons to cause the unbalanced material flow may involve the eccentricity of the holes and the unbalanced deformation of the mandrel during the extrusion process. The latter may also be induced by the eccentricity of the holes.

In the present study, the process parameters that affect the shape of the extruded tube were examined using the finite element analysis. In order to express the curvature of the extruded tube, a bending angle $(\alpha)$ is defined, as shown in Fig. 6(a). The coordinates of the end points $P_{1}$ and $P_{2}$ shown in Fig. $6(a)$ are denoted by $\left(x_{1}, y_{1}, z_{1}\right)$ and $\left(x_{2}, y_{2}, z_{2}\right)$, respectively. Since both $\mathrm{P}_{1}$ and $\mathrm{P}_{2}$ lie on the symmetric plane $x-z$, the bending angle is defined by $\alpha=\tan ^{-1}\left(\left(z_{2}-z_{1}\right) /\left(x_{2}-x_{1}\right)\right)$. A positive bending angle represents an inward bending and a negative bending angle is for an outward bending.

One of the major process variables that affect the curvature of the extruded tube is the location of holes. The location of the holes can be expressed by the eccentricity from the centre of the billet (Peng and Sheppard, 2004). Hence, the eccentricity ratio $(e)$ defined by $e=b /(a+b)$ is used to express the location of holes in the multi-hole extrusion process, where $0 \leq e<1$, and the dimensions of $a$ and $b$ are displayed in Fig. 6(b). From the definition, the eccentricity is zero for $b=0$, i.e., the hole is located at the centre, and a larger value of $b$ represents that the holes are located at a distance from the centre.

Fig. 4 - Schematic drawing for multi-hole extrusion. 


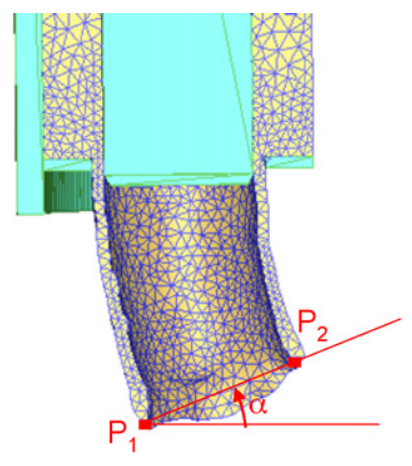

(a)

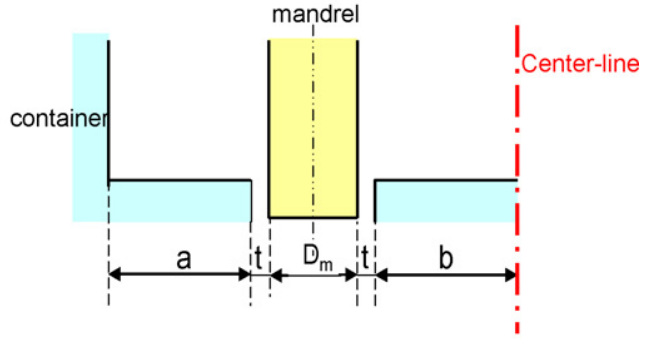

(b)

Fig. 6 - Bending angle of extruded tube and location of holes. (a) Bending angle. (b) Location of holes.

\section{Effects of process parameters}

The process parameters including extrusion speed, initial forming temperature, billet dimension, and hole location were examined in the present study using the finite element analysis. The multi-hole extrusions of two-hole, three-hole, and four-hole were simulated and the simulation results for the two-hole extrusion were discussed in this paper. The simulation parameters are the same as those adopted in the single-hole extrusion and the value of eccentricity of $e=0.5$ is used to represent the location of holes for most simulations.

The effect of the extrusion speed on the tube shape was studied first. Two extrusion speeds of $0.6 \mathrm{~mm} / \mathrm{s}$ and $1.2 \mathrm{~mm} / \mathrm{s}$ used in the two-hole extrusion were simulated. The bending angles of the extruded tubes were calculated at selected strokes during the extrusion process, and the results are shown in Fig. 7(a). As seen in Fig. 7(a), all bending angles have negative values, representing an outward bending in the extruded tubes. It is also noted in Fig. 7(a) that a higher extrusion speed results in a larger bending angle. It implies that the higher extrusion speed will enlarge the difference between the material flows on both sides of the hole and the material flow is much faster at the side close to the billet centre.

Fig. 7(b) shows the bending angles of the extruded tubes obtained from the extrusion processes with different initial extrusion temperatures of $360^{\circ} \mathrm{C}, 380^{\circ} \mathrm{C}$, and $400^{\circ} \mathrm{C}$, respectively. It is seen in Fig. 7(b) that a lower extrusion temperature will cause a larger bending angle in the extruded tube. However, the difference is not so significant and the extrusion temperature has a less influence on the shape of the extruded tube.

In order to investigate the effect of billet dimension on the shape of extruded tubes, two billets with $203.2 \mathrm{~mm}$ and $254 \mathrm{~mm}$ in outside diameter are used in the extrusion simulations. The bending angles are calculated at different stages of extrusion. It is observed that the larger billet causes less curvature in the extruded tubes. It may be because the larger billet provides more material around the mandrel and makes the material flow more balanced at the exit of extrusion die.

The material flow in the multi-hole extrusion can also be affected by the geometry of the die. A fully symmetric geometry is considered as the most desirable die design. However, for multi-hole extrusion, a fully symmetric die shape is not obtainable. The eccentric arrangement of holes may cause unbalanced flow at the exit of extrusion die. In the present study, the hole arrangements with various eccentricity ratios of $0.3,0.35,0.4$ and 0.5 were adopted to design the die geometry. The eccentricity ratio of 0.35 represents that the hole is located at the centroid of one half of the circular die. The extruded tubes obtained from the finite element simulation results are shown in Fig. 8. As seen in Fig. 8, the bending angle

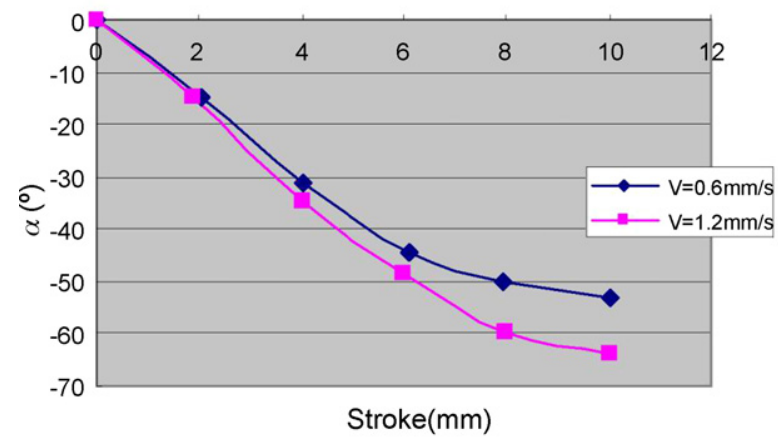

(a) various extrusion speeds.

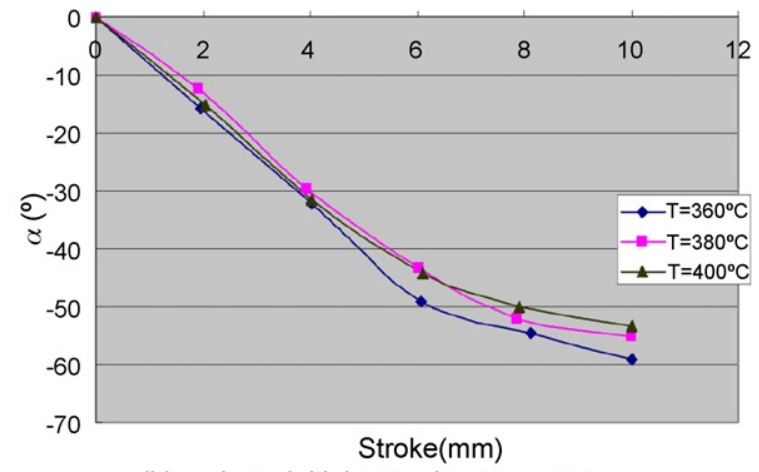

(b) various initial extrusion temperatures

Fig. 7 - Bending angles for various extrusion speeds and various initial extrusion temperatures. (a) Various extrusion speeds. (b) Various initial extrusion temperatures. 


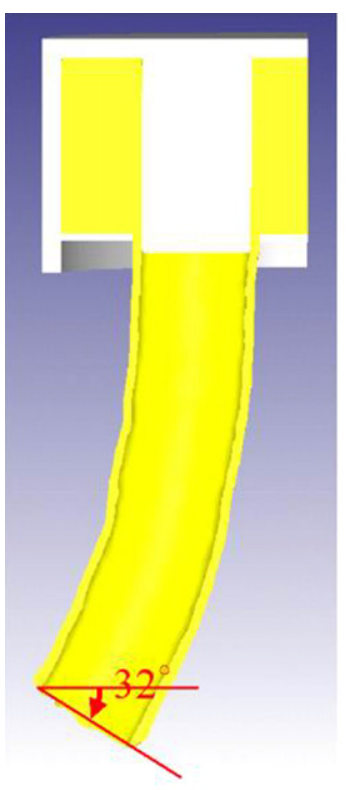

(a) $e=0.4$

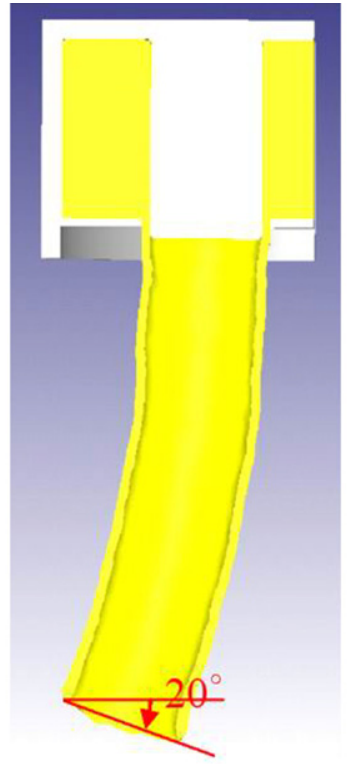

(b) $e=0.35$

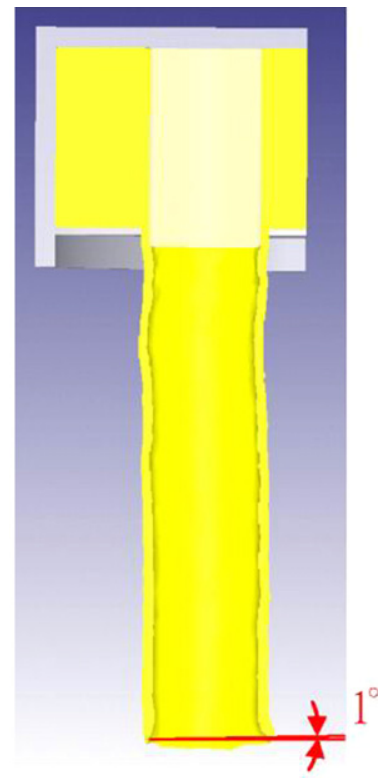

(c) $e=0.3$

Fig. 8 - Extruded tubes for various locations of holes.

decreases as the eccentricity ratio becomes smaller, i.e., the hole is closer to the centre of the die. The bending angles for various values of eccentricity ratio at different stages in the two-hole extrusion process were also examined. The simulation results reveal that when $\alpha \leq 0.3$, the sign of bending angle changes from negative to positive. It indicates that there exists an optimum eccentricity ratio that leads to a balanced flow at the exit of extrusion die, and for two-hole extrusion, $\alpha=0.3$ can be considered as an optimum value of eccentricity ratio.

\section{Concluding remarks}

In the present study, both the single-hole extrusion and the multi-hole extrusion of aluminum-alloy tubes were examined. The finite element analysis confirms that the friction has an insignificant effect on the extrusion load in an indirect extrusion process. The good agreement between the finite element simulation results and the experimental data obtained in the single-hole extrusion also validates the efficiency and the accuracy of the finite element simulations.

In the multi-hole extrusion, the curved shape of the extruded tube is the major defect produced in the extrusion process. The curved shape of the extruded tube is due to the unbalanced material flow at the exit of the extrusion die, which is caused by various process parameters. The unbalanced material flow may also result in a non-uniform deformation of the mandrel, which produces even more curvature on the extruded tubes. The finite element analysis reveals that the extruded tube may bend inward or outward, depending on the number of holes and the value of eccentricity ratio. It also found that a higher extrusion speed, or a lower extrusion temperature, or a smaller billet leads to a larger bending angle in the extruded tubes. The location of holes in an extrusion die is considered as the most crucial variable that produces curved extruded tubes. It is found that the bending angle of the extruded tube becomes smaller as the position of holes is near by the centroid of the die area. However, the finite element analysis reveals that there exists an optimum eccentricity ratio that leads to a balanced flow at the exit of extrusion die, and for the two-hole extrusion, $\alpha=0.3$ can be considered as an optimum value of eccentricity ratio.

The developed approach could be extended to other multihole extrusion of more than two tubes and help the die engineer to design a more productive extrusion process.

\section{Acknowledgments}

The authors would like to thank the Chung-Shan Institute of Science \& Technology of the Republic of China for financially supporting this research. They would also like to thank SFTC for the help in running the DEFORM program.

\section{REF E RE N C E S}

Jo, H.H., Lee, S.K., Lee, S.B., Kim, B.M., 2002. Prediction of welding pressure in the non-steady state porthole die extrusion of Al7003 tubes. Int. J. Mach. Tools Manuf. 22, 753759.

Kim, K.J., Lee, C.H., Yang, D.Y., 2002. Investigation into the improvement of welding strength in three-dimensional extrusion of tubes using porthole dies. J. Mater. Process. Technol. 130-131, 426-431.

Lee, G.A., Im, Y.T., 2002. Analysis and design of flat-die hot extrusion process. 2. Numerical design of bearing lengths. Int. J. Mech. Sci. 44, 935-946.

Lee, G.A., Kwak, D.Y., Kim, S.Y., Im, Y.T., 2002. Analysis and design of flat-die hot extrusion process. 1. Three-dimensional finite element analysis. Int. J. Mech. Sci. 44, 915-934. 
Li, L., Zhou, J., Duszczyk, J., 2004. Prediction of temperature evolution during the extrusion of 7075 aluminum-alloy at various ram speeds by means of 3D FEM simulation. J. Mater. Process. Technol. 145, 360-

370.
Peng, Z., Sheppard, T., 2004. Simulation of multi-hole die extrusion. Mater. Sci. Eng. 367, 329-342.

Ulysse, P., Johnson, R.E., 1998. A study of the effect of the process variables in unsymmetrical single-hole and multi-hole extrusion processes. J. Mater. Process. Technol. 73, 213-225. 